\title{
Computational modeling of the arterial wall based on layer-specific histological data
}

\author{
Tao Jin · Ilinca Stanciulescu
}

Received: date / Accepted: date

\begin{abstract}
Arterial walls typically have a heterogeneous structure with three different layers (intima, media and adventitia). Each layer can be modeled as a fiberreinforced material with two families of relatively stiff collagenous fibers symmetrically arranged within an isotropic soft ground matrix. In this paper, we present two different modeling approaches, the embedded fiber (EF) approach and the angular integration (AI) approach, to simulate the anisotropic behavior of individual arterial wall layers involving layer-specific data. The EF approach directly incorporates the microscopic arrangement of fibers that are synthetically generated from a random walk algorithm and captures material anisotropy at the element level of the finite element (FE) formulation. The AI approach smears fibers in the ground matrix and treats the material as homogeneous, with material anisotropy introduced at the constitutive level by enhancing the isotropic strain energy with two anisotropic terms. Both approaches include the influence of fiber dispersion introduced by fiber angular distribution (departure of individual fibers from the mean orientation), and take into consideration the dispersion caused by fiber waviness, which has not been previously considered. By comparing the numerical results with the published experimental data of different layers of a human aorta, we show that by using histological data both approaches can successfully capture the anisotropic behavior of individual arterial wall layers. Furthermore, through a comprehensive parametric study, we establish the connections between the AI phenomenological material parameters and the EF parameters having straightforward physical or geometrical interpretations. This study provides valuable insight for the calibration of phenomenological parameters used in the homogenized modeling based on the fiber microscopic arrangement. Moreover, it facilitates a better
\end{abstract}

Tao Jin

Department of Civil and Environmental Engineering, Rice University, Houston, TX 77005, USA

Ilinca Stanciulescu

Department of Civil and Environmental Engineering,

Rice University, Houston, TX 77005, USA

E-mail: ilinca.s@rice.edu 
understanding of individual arterial wall layers, which will eventually advance the study of the structure-function relationship of arterial walls as a whole.

Keywords Arterial walls · Angular integration · Embedded fiber approach

\section{Introduction}

Understanding the structure-function relationship of individual arterial wall layers provides insight into the pathophysiology of arterial wall diseases such as arteriosclerosis, allows for better predictions for the outcome of interventional treatments such as balloon angioplasty (Holzapfel et al. 2002), and advances various biomedical applications such as artificial heart valve (Durst et al. 2011; Hasan et al. 2014). In the thickness direction, arterial walls are typically separated into three layers, intima, media and adventitia (or externa), with different histology and mechanical properties. The intima is the innermost arterial layer, the pathological change (thickening and stiffening with age) of which may be associated with arteriosclerosis (Holzapfel et al. 2000, 2004). The media is the middle layer consisting of smooth muscle cells, elastin and collagen fibers. The micro-structural arrangement of this layer gives it high strength and the ability to resist loads in both the axial and circumferential directions (Gasser et al. 2006). The adventitia is the outermost layer and mainly consists of thick bundles of collagen fibrils that contribute significantly to the artery stability and strength (Holzapfel et al. 2005). For a more detailed description of the three layers, see Holzapfel et al. (2000). Although the three layers of arterial walls have very different mechanical properties, they all exhibit significant nonlinear and anisotropic behaviors. For example, according to the experimental data from a human abdominal aorta (Holzapfel 2006), the media layer is much stiffer in the circumferential direction than in the axial direction.

Uniaxial (in vitro) tension tests are widely used to study the mechanical behavior of different arterial wall layers. However, these tests are not sufficient for the construction of multi-dimensional models to predict the material behavior in physiological loading states. Conducting experiments that closely mimic the in vivo loading state would provide more valuable information, but such experiments often put higher demands on the equipment and human skills. On the other hand, developing numerical strategies involving layer-specific histological information may provide an effective and inexpensive choice to study the material behavior of different arterial wall layers under complex loading conditions.

To date, many efforts were devoted to developing constitutive models of arterial wall in the context of continuum mechanics. The pioneer effort came from Fung and co-workers (Fung 1967; Fung et al. 1979), who proposed an exponential form for the strain energy function that was widely adopted and further improved to model the mechanical behavior of arterial walls and other biological tissues (Vawter et al. 1979; Chuong and Fung 1983; Fung 1983). However, the Fung model does not include the material intrinsic structure, therefore its predictive capability is largely limited. Moreover, this type of model is proved to lose convexity for certain ranges of material parameters (Wilber and Walton 2002). Another well-known constitutive model for arterial walls was proposed by Holzapfel et al. (2000) and is known as the Holzapfel-Gasser-Ogden (HGO) model, in which an exponential strain energy function was also adopted. The HGO model includes the pseudoinvariants of structural tensors (Spencer 1984) in the anisotropic strain energy to 
consider the influence of fiber orientation on the material mechanical behavior, and can maintain important mathematical properties such as the polyconvexity condition (Balzani et al. 2006) as long as the contribution from fibers under compression is switched off. However, the HGO model assumes that all fibers are concentrated in several orientations and does not capture the influence of fiber dispersion. To address this limitation, Gasser et al. (2006) adopted the concept of generalized structural tensor (GST) to consider the statistical distribution of fibers. Despite the simple formulation and efficiency, it was later pointed out by Federico and Herzog (2008) that the GST model was only valid when all fibers were in tension and the angular fiber dispersion was small. Federico and Gasser (2010) later proposed a more general method based on the angular integration (AI) of a strain energy function containing the statistical information of fiber distributions. Although the AI approach is computationally more expensive, it is valid when the fiber angular dispersion is large and can exclude contributions from fibers under compression. For a detailed comparison between the GST and AI approaches, see Cortes et al. (2010). All these efforts belong to the homogenized modeling strategy and possess several advantages: the finite element (FE) formulations are relatively straightforward, and mathematical requirements such as polyconvexity are satisfied. However, all the models only consider the dispersion introduced by fiber angular distribution with respect to the mean orientation and fail to include the dispersion caused by fiber waviness. Moreover, the phenomenological material parameters involved do not have straightforward physical interpretations. To identify them, an inverse problem that may be ill-posed needs to be solved.

An alternative to homogenization relies on directly incorporating the microscopic arrangement of fibers in the modeling process (Lake et al. 2012; Zhang et al. 2013; Jin and Stanciulescu 2015). The fiber arrangement can either be directly obtained using image processing from real tissues (D'Amore et al. 2014; Carleton et al. 2015) or synthetically generated (Huisman et al. 2007; Barocas 2007; Sander et al. 2009; Liu et al. 2013; Heidemann et al. 2015). Although the detailed modeling strategy is more complex to implement, puts higher demand on computational resources, and often requires representative volume elements (RVEs) identification or mesh refinement analysis to eliminate size effects (Shahsavari and Picu 2013; Jin and Stanciulescu 2015), the parameters involved have physical or geometrical interpretations and can often be directly obtained from experiments.

In this paper, we present two different approaches, the embedded fiber (EF) approach belonging to the detailed modeling strategy and the angular integration (AI) approach belonging to the homogenized modeling strategy, to simulate the anisotropic behavior of individual arterial wall layers using layer-specific histological data. Both approaches take into consideration the influence of fiber dispersion introduced by fiber angular distribution (departure of individual fibers from the mean orientation) and fiber waviness. Through a comprehensive parametric study, we establish relationships between the phenomenological parameters used in the AI approach and those used in the EF approach that have straightforward physical meaning. These relationships are helpful to form a better understanding of the connection between the microscopic structure and the macro-mechanical functions of different arterial wall layers. First, the EF and AI approaches are described. Next, the method to extract modeling parameters from layer-specific histological data is presented. By comparing the numerical results with the published experimental data, we demonstrate that both approaches can successfully 
capture the anisotropic behavior of different arterial wall layers. Finally, we carry out a comprehensive parametric study and establish several relationships between the material parameters used in the two approaches.

\section{Methods}

Arterial walls are typically composed of three different layers in the thickness direction: intima, media and adventitia. Although each layer has different histology, they all can be modeled as fiber-reinforced materials with two families of collagenous fiber bundles symmetrically arranged in an isotropic soft ground matrix (Holzapfel et al. 2000; Holzapfel 2006). In this section, we first describe the embedded fiber (EF) and the angular integration (AI) modeling approaches both involving layer-specific histological data to simulate the mechanical behavior of individual arterial wall layers. Then, we use published data for a human abdominal aorta (Holzapfel 2006) as an example to demonstrate how to obtain material parameters based on experimental data. Lastly, we propose several relationships to establish the connections between the material parameters used in the two modeling approaches.

\subsection{Formulations}

The embedded fiber (EF) approach and the angular integration (AI) approach are proposed to simulate the mechanical behavior of different arterial wall layers. The EF approach directly incorporates the microscopic arrangement of fibers that are modeled as truss elements in the numerical simulation with the assumption that fiber stretching is the main deformation mode (Zhang et al. 2013; D'Amore et al. 2014). The fibers are embedded in the surrounding matrix and the two material components deform together. The material parameters used in this approach have direct physical or geometrical interpretations. The AI approach smears fibers into the matrix, thus belonging to the homogenization class of methods. Arterial wall layers typically contain two families of collagenous fiber bundles that are symmetrically arranged and embedded in the ground matrix (Holzapfel et al. 2000). The two families of fiber bundles make the material anisotropic. To consider this histology in the AI approach, the material anisotropy is introduced at the constitutive level by enhancing the isotropic strain energy that describes the contribution from the matrix component with two anisotropic terms (Gasser et al. 2006; Balzani et al. 2006), each of which contains several phenomenological material parameters and represents the contribution from one of the two families of fiber bundles. Both approaches assume that the two families of fibers are in the plane formed by the circumferential and axial directions, symmetrically arranged with respect to the circumferential direction, and have the same mechanical properties.

\subsubsection{Embedded fiber approach}

In the embedded fiber (EF) approach, a random walk algorithm (Spanos and Esteva 2009; Jin and Stanciulescu 2015) is implemented to generate the fiber microscopic arrangement based on the following assumptions: 
1. Each fiber is piecewise linear and formed by a fixed number of segments $N_{\text {seg }}$, and each segment has length $l_{\text {seg }}$;

2. For the family of fibers forming the mean orientation $\phi$ with respect to the circumferential direction, the initial angle $\theta_{0}$ of each fiber follows the $\pi$-periodic Von Mises (VM) distribution with the Von Mises parameter $b_{0}$ (Gasser et al. 2006; Wang et al. 2012). The probability density function (PDF) $\rho\left(\theta, \phi, b_{0}\right)$ of the VM distribution is expressed as follows

$$
\rho\left(\theta, \phi, b_{0}\right)=\frac{e^{b_{0} \cos 2(\theta-\phi)}}{\pi I_{0}\left(b_{0}\right)}, \quad I_{0}\left(b_{0}\right)=\frac{1}{\pi} \int_{0}^{\pi} e^{b_{0} \cos \theta} \mathrm{d} \theta,
$$

where $I_{0}\left(b_{0}\right)$ denotes the modified Bessel function of the first kind of order zero. Eq. 1 satisfies the normalization condition $\int_{-\pi / 2}^{\pi / 2} \rho\left(\theta, \phi, b_{0}\right) \mathrm{d} \theta=1$. The influence of $b_{0}$ on the Von Mises distribution is shown in Fig. 1;

3. The relative angle $\theta_{r}$ between two neighboring segments in the same fiber follows a uniform distribution, $\theta_{r} \sim \operatorname{unif}\left(-\theta_{\max }, \theta_{\max }\right)$;

4. The total number of fibers in the material is decided by the fiber volume fraction $\mu$.

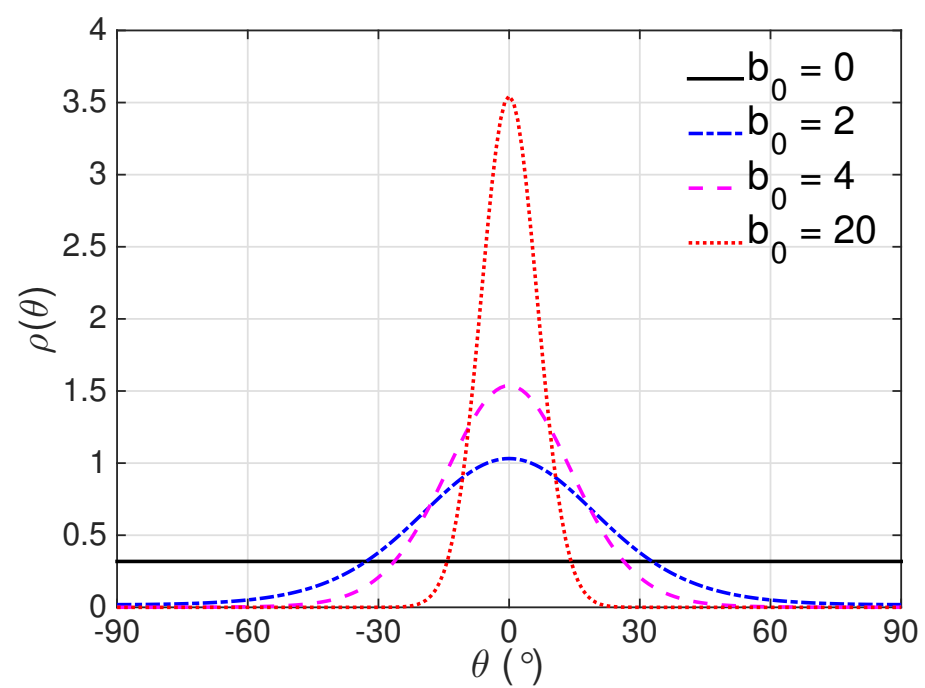

Fig. 1 Influence of the parameter $b_{0}$ on the Von Mises distribution with the mean orientation $\phi=0^{\circ}$

To generate the family of fibers with the mean orientation $\phi$ (with respect to the circumferential direction), the main steps of the algorithm are summarized as follows:

1. Specify a $2 \mathrm{D}$ area with thickness $t$;

2. Select a random point inside the area as the starting point of a fiber chain;

3. Choose an initial angle $\theta_{0} \sim \operatorname{VM}\left(\phi, b_{0}\right)$ and from the starting point generate the first segment with length $l_{\text {seg }}$;

4. Generate the next connected segment with an angle $\theta_{r} \sim \operatorname{unif}\left(-\theta_{\max }, \theta_{\max }\right)$ 
relative to the previous segment;

5 . Repeat Step 4 until the fiber is out of the area or the fixed number of segments $N_{\text {seg }}$ is reached;

6 . Repeat Steps 2 to 5 until the assigned fiber volume fraction $\mu$ is reached. Following the same Steps 2 to 6 , the family of fibers with the mean orientation $-\phi$ is generated.

Figure 2 is a schematic representation of the geometrical parameters used in the random walk algorithm. The fiber dispersion has two different causes, departure of individual fibers from the mean orientation measured by the initial angle $\theta_{0} \sim \operatorname{VM}\left(\phi, b_{0}\right)$, and the fiber waviness represented by the relative angle $\theta_{r} \sim \operatorname{unif}\left(-\theta_{\max }, \theta_{\max }\right)$. The level of fiber dispersion increases as $\theta_{\max }$ increases, indicating larger waviness (Fig. 3), and decreases as $b_{0}$ increases, indicating that fibers are more concentrated toward the mean orientation.

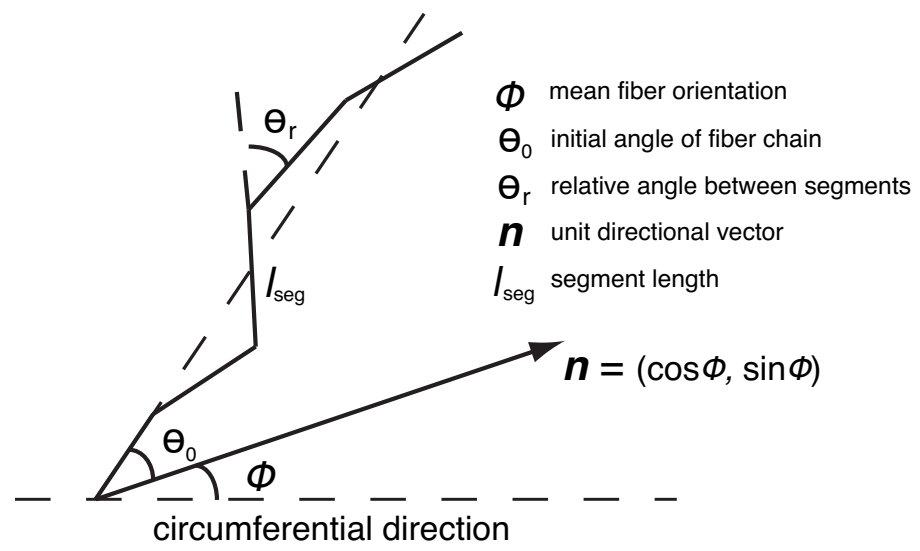

Fig. 2 Geometric interpretations of the variables used in the random walk algorithm

In order to directly incorporate the generated fiber microscopic structure into the numerical simulation, the embedded fiber approach is adopted. This approach assumes that fibers are embedded in the ground matrix and the two material components deform together, thus is essentially equivalent to the affine fiber kinematics (Lanir 1983; MacKintosh et al. 1995; Agoram and Barocas 2001; Storm et al. 2005). The affine fiber kinematics is shown to be a valid assumption for many biological tissues including pericardial collagenous tissues (Fan and Sacks 2014)

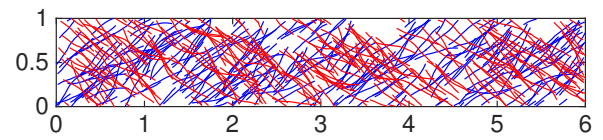

(a) $\theta_{\max }=10^{\circ}$

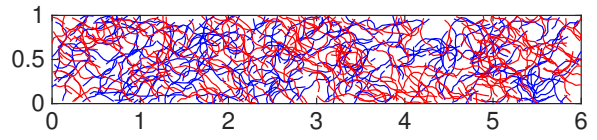

(b) $\theta_{\max }=45^{\circ}$

Fig. 3 Influence of the relative angle between segments $\theta_{r} \sim \operatorname{unif}\left(-\theta_{\max }, \theta_{\max }\right)$ on the level of fiber dispersion introduced by fiber waviness 
and mitral valve anterior leaflet (Lee et al. 2015). Figure 4 shows a rectangular arterial wall layer sample with two families of fibers synthetically generated by the random walk algorithm. After the material sample is meshed, each fiber segment is either completely inside one element or crosses element boundaries. For the latter case, the coordinates of the intersection points are calculated and divide the segment into multiple sub-segments. The Cartesian coordinates of the intersection points formed by fibers and element boundaries, combined with the two endpoints of all fiber segments, are recorded in a data list $\mathrm{L}=\left\{\left(x_{i}, y_{i}\right), i=1,2,3, \cdots\right\}$. In the isoparametric element, the Cartesian coordinates $\boldsymbol{x}$ and the natural coordinates $\boldsymbol{\xi}$ have the following relationship

$$
\boldsymbol{x}(\boldsymbol{\xi})=N_{a}(\boldsymbol{\xi}) \boldsymbol{x}_{a}^{(e)},
$$

where subscript $a$ is the element nodal number, $\boldsymbol{x}_{a}^{(e)}$ are the nodal coordinates for the $e$-th element, and $N_{a}$ is the element interpolation function associated with node $a$. Here, the Einstein summation convention is used. Through Newton-Raphson iterations, the natural coordinates $\boldsymbol{\xi}$ corresponding to the Cartesian coordinates $\boldsymbol{x}$ in the list $\mathrm{L}$ are calculated and stored in a data structure shown in Table 1 . The $i$-th row of this data structure records the natural coordinates of the start $\left(\xi_{1}, \eta_{1}\right)$ and the end $\left(\xi_{2}, \eta_{2}\right)$ of each fiber segment contained in the $i$-th element. Note that different elements do not necessarily have the same number of fiber segments. This data structure provides all the geometric information of the microscopic structure of fibers needed for the finite element simulation.

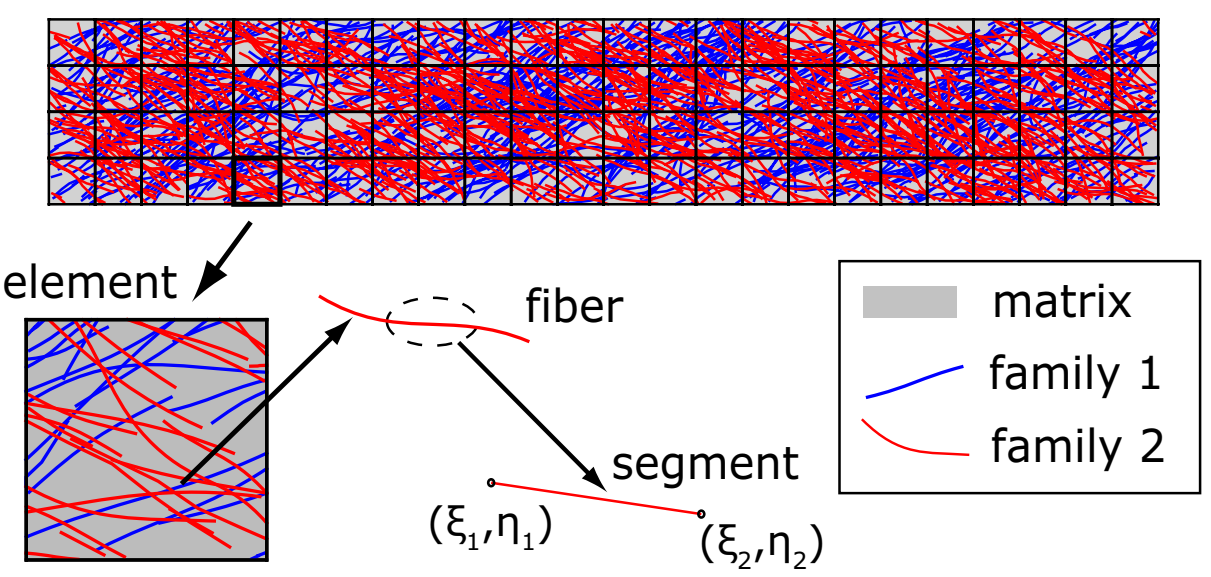

Fig. 4 Synthetically generated arterial wall layer sample meshed with four-node quad element (the mean orientations of the two families of fibers $\phi= \pm 30^{\circ}$, and the Von Mises parameter $b_{0}=10.0$ )

The two symmetrically arranged families of fibers have the same material properties, and the mechanical property of individual fiber segments is expressed by 
Table 1 Data structure constructed to record the natural coordinates of the fiber segments contained inside each finite element

\begin{tabular}{c|cccc}
\hline \hline Elmt No. & $\left(\xi_{1}, \eta_{1}\right),\left(\xi_{2}, \eta_{2}\right)$ & $\left(\xi_{1}, \eta_{1}\right),\left(\xi_{2}, \eta_{2}\right)$ & $\cdots$ & $\left(\xi_{1}, \eta_{1}\right),\left(\xi_{2}, \eta_{2}\right)$ \\
\hline 1 & 1st segment & 2nd segment & $\cdots$ & last segment \\
\hline 2 & 1st segment & 2nd segment & $\cdots$ & last segment \\
\hline 3 & 1st segment & 2nd segment & $\cdots$ & last segment \\
\hline$\vdots$ & $\vdots$ & $\vdots$ & $\vdots$ & $\vdots$ \\
\hline $\mathrm{n}$ & 1st segment & 2nd segment & $\cdots$ & last segment \\
\hline \hline
\end{tabular}

an exponential form of strain energy function

$$
\Psi_{f}(\lambda)=\left\{\begin{array}{cc}
\frac{1}{2 z_{f}} k_{f} L_{0}^{2}\left(e^{z_{f}\left(\lambda^{2}-1\right)^{2}}-1\right) & \lambda \geq 1 \\
0 & \lambda<1
\end{array}\right.
$$

where $k_{f}$ is the axial elastic modulus of fiber segment, $z_{f}$ is a dimensionless parameter controlling the fiber nonlinearity, $L_{0}$ is the segment original length, and $\lambda$ is the stretch ratio. It can be verified that $\left.\Psi_{f}(\lambda)\right|_{\lambda=1}=0$ and $\partial \Psi_{f}(\lambda) /\left.\partial \lambda\right|_{\lambda=1}=0$, indicating the stress-free state when no deformation occurs. This form of fiber strain energy functions is consistent with the widely accepted assumption that fibers can only sustain tensile force, but are not able to resist compression (Holzapfel et al. 2000; Balzani et al. 2006; Pandolfi and Vasta 2012).

The ground matrix material is typically described by an isotropic constitutive model such as the Yeoh model (Sarma et al. 2003; Martins et al. 2006; D'Amore et al. 2014) or the neo-Hookean model (Holzapfel et al. 2000; Gasser et al. 2006; Balzani et al. 2006; Jin and Stanciulescu 2015). Here, we adopt the modified neo-Hookean model with the volumetric term serving as a penalty to ensure the material nearly incompressibility

$$
\Psi_{m}(\boldsymbol{F})=\frac{1}{4} K\left(J^{2}-1-2 \ln J\right)+\frac{1}{2} G\left(\overline{I_{1}}-3\right),
$$

where $K$ is the bulk modulus, $G$ is the shear modulus, $J=\operatorname{det} \boldsymbol{F}$ is the determinant of the deformation gradient tensor $\boldsymbol{F}$, and $\bar{I}_{1}=J^{-\frac{2}{3}} \operatorname{tr}\left(\boldsymbol{F}^{\mathrm{T}} \boldsymbol{F}\right)$ is the modified tensor invariant.

In the displacement formulation, the primary unknowns are element nodal displacements $\boldsymbol{u}$. The total strain energy $\Pi(\boldsymbol{u})$ is decoupled into the contributions from the ground matrix and the fibers. After spatial discretization, $\Pi(\boldsymbol{u})$ can be written as

$$
\Pi(\boldsymbol{u})=\sum_{e=1}^{N_{e l}}\left[\int_{\Omega_{0}^{(e)}} \Psi_{m}(\boldsymbol{F}(\boldsymbol{u})) \mathrm{d} \Omega+\sum_{i=1}^{N^{(e)}} \Psi_{f}\left(\lambda^{(i)}(\boldsymbol{u})\right)\right],
$$

where $\Psi_{f}$ is the fiber strain energy and takes the exponential form (Eq. 3), $\Psi_{m}$ is the matrix strain energy (Eq. 4), $\lambda^{(i)}$ is the stretch ratio of the $i$-th fiber segment, $N_{e l}$ is the total number of finite elements in the discretized system, $\Omega_{0}^{(e)}$ is the spatial domain occupied by the $e$-th element in the reference configuration, and 
$N^{(e)}$ is the number of fiber segments contained inside the $e$-th element. For an arbitrary fiber segment with the stretch ratio $\lambda$, the component form of its contribution to the element residual $\mathbf{R}_{f}^{(e)}$ and the stiffness matrix $\mathbf{K}_{f}^{(e)}$ are derived from a variational approach and can be written as follows

$$
R_{f, i A}^{(e)}=2 k_{f} e^{z_{f}\left(\lambda^{2}-1\right)^{2}}\left(\lambda^{2}-1\right)\left(x_{i}^{(2)}-x_{i}^{(1)}\right)\left(N_{A}\left(\boldsymbol{\xi}^{(2)}\right)-N_{A}\left(\boldsymbol{\xi}^{(1)}\right)\right)
$$

and

$$
\begin{aligned}
K_{f, A i B j}^{(e)}= & 2 k_{f} e^{z_{f}\left(\lambda^{2}-1\right)^{2}}\left(\lambda^{2}-1\right) \\
& \left(N_{A}\left(\boldsymbol{\xi}^{(2)}\right)-N_{A}\left(\boldsymbol{\xi}^{(1)}\right)\right) \\
& \left(N_{B}\left(\boldsymbol{\xi}^{(2)}\right)-N_{B}\left(\boldsymbol{\xi}^{(1)}\right)\right) \delta_{i j} \\
+ & \frac{4 k_{f}}{L_{0}^{2}} e^{z_{f}\left(\lambda^{2}-1\right)^{2}}\left[1+2 z_{f}\left(\lambda^{2}-1\right)^{2}\right] \\
& {\left[\left(x_{i}^{(2)}-x_{i}^{(1)}\right)\left(N_{A}\left(\boldsymbol{\xi}^{(2)}\right)-N_{A}\left(\boldsymbol{\xi}^{(1)}\right)\right)\right] } \\
& {\left[\left(x_{j}^{(2)}-x_{j}^{(1)}\right)\left(N_{B}\left(\boldsymbol{\xi}^{(2)}\right)-N_{B}\left(\boldsymbol{\xi}^{(1)}\right)\right)\right] }
\end{aligned}
$$

where the superscripts (1) and (2) represent the two ends of the fiber segment, $A$, $B$ are the element nodal numbers, $i, j$ are the Cartesian directions, $\boldsymbol{\xi}^{(1)}$ and $\boldsymbol{\xi}^{(2)}$ are the natural coordinates of the fiber segment under consideration (available from Table 1), and $N_{A}$ is the element interpolation function associated with node $A$. Notice that in Jin and Stanciulescu (2015), a slightly different fiber strain energy was used. Therefore, while the variational principles are the same, the counterparts of Eq. 6 and 7 are different. The total element residual $\mathbf{R}^{(e)}$ and the stiffness matrix $\mathbf{K}^{(e)}$ are the sum of the contributions from the ground matrix material and all fiber segments contained in the corresponding element

$$
\begin{aligned}
& \mathbf{R}^{(e)}=\mathbf{R}_{m}^{(e)}+\sum_{i} \mathbf{R}_{f_{i}}^{(e)}, \\
& \mathbf{K}^{(e)}=\mathbf{K}_{m}^{(e)}+\sum_{i} \mathbf{K}_{f_{i}}^{(e)} .
\end{aligned}
$$

The derivations of $\mathbf{R}_{m}^{(e)}$ and $\mathbf{K}_{m}^{(e)}$, representing the contributions from the ground matrix, can be found in most nonlinear FEM textbooks (e.g., Bonet and Wood (2008)).

In summary, the embedded fiber (EF) approach directly incorporates the microscopic arrangement of fibers in the finite element formulation at the element level. The fiber dispersion introduced by departure of individual fibers from the mean orientation is considered by the initial angle $\theta_{0} \sim \operatorname{VM}\left( \pm \phi, b_{0}\right)$ of each fiber chain, and the dispersion caused by fiber waviness is considered by the relative angle $\theta_{r} \sim \operatorname{unif}\left(-\theta_{\max }, \theta_{\max }\right)$ between neighbouring segments. The influences of the geometric parameters $\phi, b_{0}$ and $\theta_{\max }$ are implicitly included in the terms $\mathbf{R}_{f_{i}}^{(e)}$ and $\mathbf{K}_{f_{i}}^{(e)}$ representing the contribution from the fiber component. The material parameters adopted in this approach have straightforward geometrical or mechanical interpretations. 


\subsubsection{Angular integration approach}

The angular integration (AI) approach follows a homogenization methodology that smears the two families of fibers into the ground matrix and treats the material as homogeneous. In the finite element formulation, the anisotropy introduced by fibers is considered at the constitutive level. The total strain energy is decoupled into two parts, the isotropic part representing the contribution from the ground matrix $\Psi_{m}$ enhanced by the anisotropic terms $\Psi_{f}$ representing the contributions from the two families of fibers,

$$
\Psi=\Psi_{m}+\Psi_{f}(\phi)+\Psi_{f}(-\phi),
$$

where $\Psi_{m}$ is described by the modified neo-Hookean model with the strain energy function as shown in Eq. 4. The contribution from the fiber component $\Psi_{f}$ takes the following angular integration (AI) form

$$
\Psi_{f}( \pm \phi)=\int_{-\pi / 2}^{\pi / 2} \rho(\theta, \pm \phi, b) \Psi\left(I_{4}(\theta)\right) \mathcal{H}\left(I_{4}(\theta)-1\right) \mathrm{d} \theta
$$

In Eq. 11, $\Psi\left(I_{4}(\theta)\right)$ is the strain energy function representing the contribution from fibers forming an angle $\theta$ with respect to the circumferential direction, and adopts the exponential form (Fung 1967; Holzapfel et al. 2000; Balzani et al. 2006)

$$
\Psi\left(I_{4}(\theta)\right)=\frac{k_{1}}{2 k_{2}}\left[e^{k_{2}\left(I_{4}(\theta)-1\right)^{2}}-1\right],
$$

where $k_{1}$ is a stress-like parameter, $k_{2}$ is a dimensionless parameter, and $I_{4}$ is the pseudo-invariant of the structural tensor $\boldsymbol{A}$ corresponding to the unit direction $\boldsymbol{N}=(\cos \theta, \sin \theta, 0)$ in the reference configuration, defined as

$$
I_{4}=\mathbf{C}: \boldsymbol{A}=\mathbf{C}: \boldsymbol{N} \otimes \boldsymbol{N}=\boldsymbol{n} \cdot \boldsymbol{n}, \quad \boldsymbol{n}=\boldsymbol{F N} .
$$

$\mathcal{H}$ is the Heaviside function

$$
\mathcal{H}\left(I_{4}(\theta)-1\right)= \begin{cases}1 & I_{4}(\theta) \geq 1 \\ 0 & I_{4}(\theta)<1\end{cases}
$$

indicating that only the contribution from fibers under tension $\left(I_{4} \geq 1\right)$ is included in the anisotropic (fiber) strain energy. $\rho(\theta, \pm \phi, b)$ is the probability density function (PDF) of the $\pi$-periodic Von Mises distribution. Here, the Von Mises parameter $b$ that describes the level of fiber dispersion is decoupled into two parts

$$
b=b_{0}+b^{c o r}
$$

where $b_{0}$ measures the dispersion introduced by departure of individual fibers from the mean orientation $\pm \phi$, and $b^{\text {cor }}$ measures the dispersion caused by fiber waviness.

Comment: In the existing methods of the homogenized modeling, all fibers are assumed to be straight. Therefore, only the dispersion caused by departure of individual fibers from the mean orientation, measured by $b_{0}$, is included. Here, we introduce the correction term $b^{\text {cor }}$ that is treated as an extra phenomenological parameter to describe the dispersion caused by fiber waviness. 
Since the pseudo-invariant $I_{4}$ depends on the deformation state described by the deformation gradient $\boldsymbol{F}$, it is very difficult, if not impossible, to obtain the analytical expression of the integral shown in Eq. 11. Consequently, a quadrature rule is needed to numerically evaluate the integral. Here, the trapezoidal rule is applied. The integration interval $[-\pi / 2, \pi / 2]$ is divided equally into $N$ subintervals. For an arbitrary sub-interval $\left[\theta_{i}, \theta_{i+1}\right]$, the midpoint $\theta_{i+\frac{1}{2}}$ is chosen to evaluate the pseudo-invariant $I_{4}$. Therefore, the integral in Eq. 11 is approximated as

$$
\Psi_{f}( \pm \phi) \approx \sum_{i=1}^{N} \rho\left(\theta_{i+\frac{1}{2}}, \pm \phi, b\right) \Psi\left(I_{4}\left(\theta_{i+\frac{1}{2}}\right)\right) \mathcal{H}\left(I_{4}\left(\theta_{i+\frac{1}{2}}\right)-1\right) \Delta \theta_{i}, \quad \Delta \theta_{i}=\theta_{i+1}-\theta_{i} .
$$

Based on Eq. 16, the 2nd Piola-Kirchhoff stress and the material elasticity tensor in the reference configuration are derived as follows

$$
\begin{aligned}
\boldsymbol{S}_{f}( \pm \phi) & =2 \frac{\partial \Psi_{f}( \pm \phi)}{\partial \boldsymbol{C}} \\
& =\sum_{j} 2 k_{1}\left(I_{4}\left(\theta_{j+\frac{1}{2}}\right)-1\right) e^{k_{2}\left(I_{4}\left(\theta_{j+\frac{1}{2}}\right)-1\right)^{2}} \rho\left(\theta_{j+\frac{1}{2}}, \pm \phi, b\right) \Delta \theta_{j} \boldsymbol{A}\left(\theta_{j+\frac{1}{2}}\right)
\end{aligned}
$$

and

$$
\begin{aligned}
\mathbb{C}_{f}( \pm \phi)= & 2 \frac{\partial \boldsymbol{S}_{f}( \pm \phi)}{\partial \boldsymbol{C}} \\
= & \sum_{j} 4 k_{1}\left[1+2 k_{2}\left(I_{4}\left(\theta_{j+\frac{1}{2}}\right)-1\right)^{2}\right] e^{k_{2}\left(I_{4}\left(\theta_{j+\frac{1}{2}}\right)-1\right)^{2}} \rho\left(\theta_{j+\frac{1}{2}}, \pm \phi, b\right) \Delta \theta_{j} \\
& \boldsymbol{A}\left(\theta_{j+\frac{1}{2}}\right) \otimes \boldsymbol{A}\left(\theta_{j+\frac{1}{2}}\right),
\end{aligned}
$$

where $j \in\left\{1, \ldots, N \mid I_{4}\left(\theta_{j+\frac{1}{2}}\right)>1\right\}$.

Comment: From Fig. 1 it can be seen that the probability density $\rho(\theta)$ of the Von Mises distribution decreases rapidly to zero when the fiber angle $\theta$ is far away from the mean value. Therefore, using the full integration interval $[-\pi / 2, \pi / 2]$ to evaluate Eq. 11 may not be necessary. In the implementation, a threshold value $\epsilon=10^{-9}$ is chosen and the integration interval is reduced to $[a, b]$, so that $\rho(a)=$ $\rho(b)=\epsilon$ and $\forall \theta \in[a, b], \rho(\theta) \geq \epsilon$. Then, a quadrature rule is performed in the interval $[a, b]$, similar to Federico and Gasser (2010).

For the arterial wall layer in which the mean angles of the two symmetrical families of fibers are $\phi$ and $-\phi$ with respect to the circumferential direction, the total 2nd Piola-Kirchhoff stress and the material elasticity tensor are the sum of the contributions from the matrix and fibers

$$
\boldsymbol{S}=\boldsymbol{S}_{m}+\boldsymbol{S}_{f}(\phi)+\boldsymbol{S}_{f}(-\phi)
$$

and

$$
\mathbb{C}=\mathbb{C}_{m}+\mathbb{C}_{f}(\phi)+\mathbb{C}_{f}(-\phi),
$$

where $\pm \phi$ can be obtained from layer-specific histological images (Holzapfel 2006). The derivations of the 2nd Piola-Kirchhoff stress $\boldsymbol{S}_{m}$ and the material elasticity 
tensor $\mathbb{C}_{m}$ from the isotropic (matrix) strain energy can be found in most nonlinear FEM textbooks, for example, see (Holzapfel 2000; Bonet and Wood 2008).

In summary, the AI approach belongs to the homogenization modeling technique that smears the fiber component into the ground matrix and treats the whole material as homogeneous (Fung 1967; Holzapfel et al. 2000; Federico and Gasser 2010). The FE formulation based on this technique is very straightforward, and the computational cost is relatively low. In the AI approach, the fiber dispersion caused by departure of individual fibers from the mean orientation is considered by the Von Mises parameter $b_{0}$, and the dispersion caused by fiber waviness is considered in this approach by introducing the correction term $b^{\text {cor }}$. The FE formulation captures the anisotropy at the constitutive level with several phenomenological material parameters.

Comment: In the EF approach, the expressions of the element residual $\mathbf{R}_{f}^{(e)}$ and the stiffness matrix $\mathbf{K}_{f}^{(e)}$ depend on the specific form of the strain energy function. In Jin and Stanciulescu (2015), the fiber strain energy function is

$$
\Psi_{f}(\lambda)=\left\{\begin{array}{cc}
\frac{1}{2 z_{f}} k_{f} L_{0}^{2}\left(e^{z_{f}(\lambda-1)^{2}}-1\right) & \lambda \geq 1 \\
0 & \lambda<1 .
\end{array}\right.
$$

Notice the only difference between the above strain energy function and the one adopted in this work (Eq. 3) is that the former uses $\lambda$ in the exponential term, while the latter uses $\lambda^{2}$. The rationale behind this model evolution comes from the strain energy function Eq. 12 used in the AI approach. Recall that $I_{4}=\lambda^{2}$. Since we aim to establish the equivalence between the EF and AI approaches, we decide to adopt $\lambda^{2}$ in the exponential term (Eq. 3) that changes the expressions of $\mathbf{R}_{f}^{(e)}$ and $\mathbf{K}_{f}^{(e)}$.

\subsection{Parameter identification}

Holzapfel (2006) conducted a comprehensive experimental study on the mechanical properties of different layers (intima, media and adventitia) of a human abdominal aorta and obtained the layer-specific histological data of fiber bundles. We use this experiment as an example to demonstrate how to obtain the material parameters based on experimental data. In the experiment, from each arterial layer two rectangular samples with the axial and circumferential orientations are cut out (Fig. 5). The ratio between the length and width of each sample is around six so that the desired homogeneous stress-strain state could be achieved during the test. Table 2 shows the mean fiber angles and standard deviations (SD) with respect to the circumferential direction of different layers. For the histological images, interested readers are referred to Fig. 4 in Holzapfel (2006). Recall that the two families of fibers in each arterial wall layer are symmetrically arranged and assumed to follow the $\pi$-periodic Von Mises distribution $\operatorname{VM}\left(\phi, b_{0}\right)$ with the parameter $b_{0}$ capturing the level of dispersion introduced by the departure of fibers from the mean orientations $\pm \phi$. The standard deviation of the Von Mises distribution (SD) and the parameter $b_{0}$ are related by

$$
\operatorname{SD}\left(b_{0}\right)=\int_{-\pi / 2}^{\pi / 2} \rho\left(\theta, \phi, b_{0}\right)(\phi-\theta)^{2} \mathrm{~d} \theta=\int_{-\pi / 2}^{\pi / 2} \frac{e^{b_{0} \cos 2(\theta-\phi)}}{\pi I_{0}\left(b_{0}\right)}(\phi-\theta)^{2} \mathrm{~d} \theta,
$$


where $\phi$ is the mean fiber angle. According to Eq. 21, a one to one relationship between SD and $b_{0}$ is established (Fig. 6), based on which the layer-specfic value $b_{0}$ (Table 2) is obtained. Notice that because of the $\pi$-periodicity, $b_{0}$ is independent of the mean fiber angle $\phi$. Consequently, the unit directional vectors presenting the mean orientations of the two families of fibers $\boldsymbol{n}_{1,2}=(\cos ( \pm \phi), \sin ( \pm \phi), 0)$ and the PDF of the Von Mises distribution $\rho\left(\theta, \pm \phi, b_{0}\right)$ followed by the initial angle $\theta_{0}$ of each fiber chain are completely decided by the histological data (Table 2 ) from the experiment.

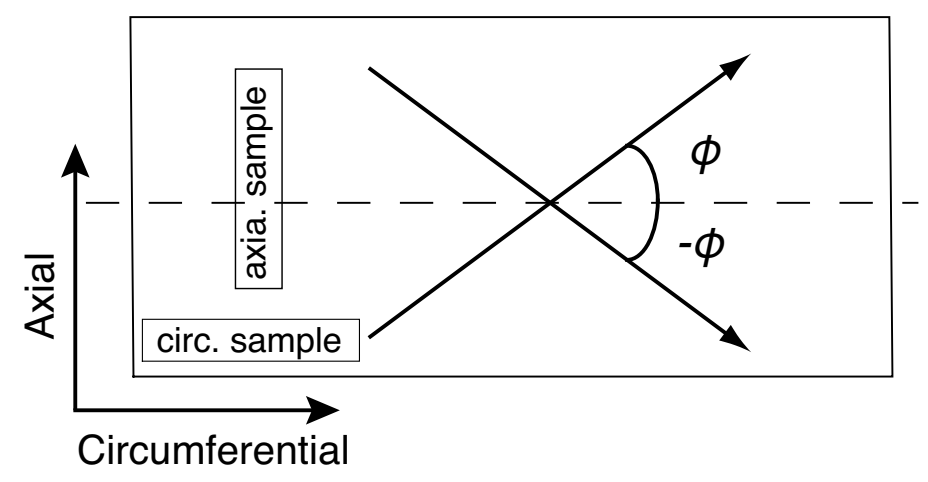

Fig. 5 Circumferential and axial samples cut from the same material specimen

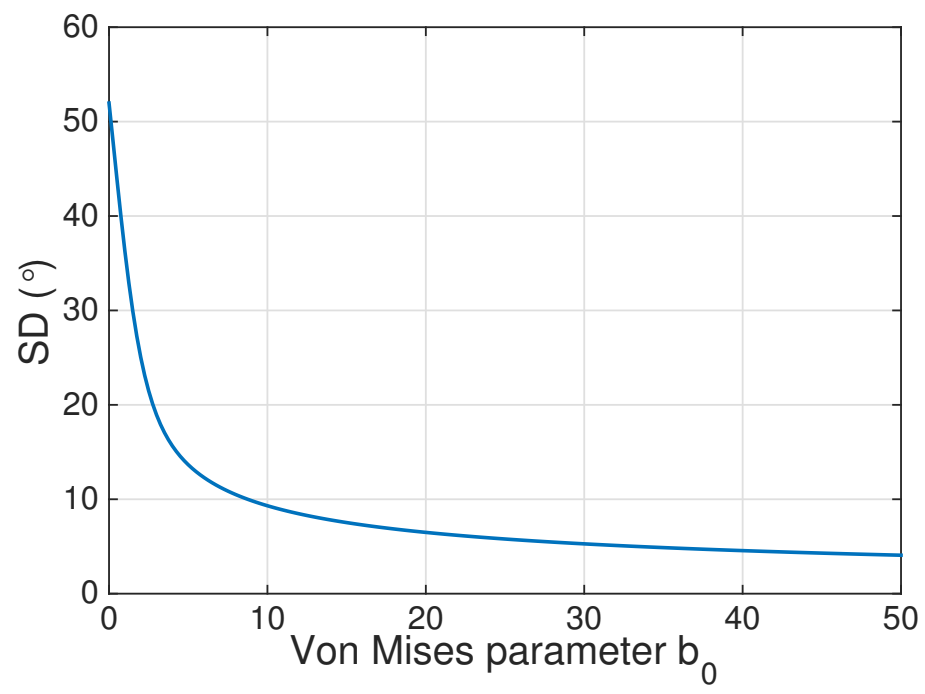

Fig. 6 Relationship between the standard deviation (SD) of fiber angles and the Von Mises parameter $b_{0}$ 
Table 2 Fiber angles (mean $\pm \mathrm{SD}$ ) with respect to the circumferential direction in different layers of the abdominal aorta and the corresponding Von Mises parameter $b_{0}$

\begin{tabular}{c|c|c|c}
\hline \hline & Intima & Media & Adventitia \\
\hline mean angle $(\phi) \pm \mathrm{SD}$ & $18.8^{\circ} \pm 8.2^{\circ}$ & $37.8^{\circ} \pm 20.6^{\circ}$ & $58.9^{\circ} \pm 14.8^{\circ}$ \\
\hline Von Mises parameter $b_{0}$ & 12.732 & 2.661 & 4.376 \\
\hline \hline
\end{tabular}

In the embedded fiber approach, the Young's modulus $E$ of the matrix material, the material parameters of individual fiber segments $k_{f}$ and $z_{f}$, and the relative angle bound $\theta_{\max }$ reflecting fiber waviness are obtained via an optimization process

$\left(E, k_{f}, z_{f}, \theta_{\max }\right)=\underset{E, k_{f}, z_{f}, \theta_{\max }}{\arg \min } \sum_{i=1}^{n}\left(\left\|\sigma^{\mathrm{circ}}\left(\lambda^{(i)}\right)-\sigma\left(\lambda^{(i)}\right)\right\|_{2}+\left\|\sigma^{\mathrm{axia}}\left(\lambda^{(i)}\right)-\sigma\left(\lambda^{(i)}\right)\right\|_{2}\right)$,

where $\sigma^{\text {circ }}$ and $\sigma^{\text {axia }}$ are the Cauchy stresses obtained from the FE simulations of the circumferential and axial samples under uniaxial tension, $\sigma\left(\lambda^{(i)}\right)$ is the corresponding experimental data, $\lambda^{(i)}$ is the stretch ratio, and $n$ is the number of experimental data records.

In the angular integration approach, the same value of $E$ obtained from Eq. 22 is used for the isotropic matrix. The phenomenological parameters $k_{1}$ and $k_{2}$ in the anisotropic strain energy and the correction of the Von Mises distribution $b^{\text {cor }}$ reflecting the dispersion caused by fiber waviness are obtained from a similar optimization process

$$
\left(k_{1}, k_{2}, b^{\text {cor }}\right)=\underset{k_{1}, k_{2}, b^{\text {cor }}}{\arg \min } \sum_{i=1}^{n}\left(\left\|\sigma^{\text {circ }}\left(\lambda^{(i)}\right)-\sigma\left(\lambda^{(i)}\right)\right\|_{2}+\left\|\sigma^{\text {axia }}\left(\lambda^{(i)}\right)-\sigma\left(\lambda^{(i)}\right)\right\|_{2}\right) .
$$

\subsection{Material parameter relationships}

Table 3 shows the comparison of the material parameters used in the embedded fiber (EF) approach and the angular integration (AI) approach. For the fiber microscopic arrangement, both approaches assume that the initial angle of individual fibers follows the $\pi$-periodic Von Mises distribution $\operatorname{VM}\left( \pm \phi, b_{0}\right)$, in which the mean fiber orientations $\pm \phi$ and the Von Mises parameter $b_{0}$ reflecting the dispersion introduced by fiber angular distribution are directly obtained from histological data. In the EF approach, the dispersion caused by fiber waviness is included via the bound $\theta_{\max }$ of the relative angle $\theta_{r}$ between neighboring segments. While in the AI approach, this type of dispersion is introduced via a correction term $b^{\text {cor }}$. For the mechanical properties, the EF approach directly includes fibers in the simulation by modeling them as truss elements with axial stiffness $k_{f}$ and the dimensionless parameter $z_{f}$ reflecting the nonlinearity. While the AI approach considers the influence of fibers through the anisotropic strain energy function in terms of the phenomenological parameters $k_{1}$ and $k_{2}$. 
Table 3 Comparison of the material parameters used in the embedded fiber and the angular integration approaches

\begin{tabular}{c|c|c}
\hline \hline & AI & EF \\
\hline FEM strategy & Constitutive level & Element level \\
\hline Mean orientation & $\pm \phi$ & $\pm \phi$ \\
\hline Dispersion (angular distribution) & $b_{0}$ & $b_{0}$ \\
\hline Dispersion (waviness) & $b^{\text {cor }}$ & $\theta_{\max }$ \\
\hline Von Mises distribution & $\rho\left(\theta, \pm \phi, b_{0}\right)$ & $\rho\left(\theta, \pm \phi, b_{0}\right)$ \\
\hline Fiber volume fraction & $k_{1}$ & $\mu$ \\
\hline Fiber stiffness & $k_{2}$ & $k_{f}$ \\
\hline Dimensionless parameter & & $z_{f}$ \\
\hline \hline
\end{tabular}

Notice that the fiber strain energy (Eq. 3) used in the EF approach and the anisotropic strain energy (Eq. 12) used in the AI approach adopt the same exponential form. Also, taking into consideration the underlying affine fiber kinematics, the following relationships between the phenomenological parameters adopted in the AI approach and those with direct physical or geometrical meaning in the EF approach are proposed

$$
\begin{gathered}
k_{1}=C k_{f} \mu \\
k_{2}=z_{f}
\end{gathered}
$$

and

$$
b^{c o r}=b^{\text {cor }}\left(b_{0}, \theta_{\max }\right),\left.\quad b^{\text {cor }}\right|_{\theta_{\max }=0}=0 .
$$

Eq. 24 suggests that the stress-like parameter $k_{1}$ in the AI approach is proportional to the fiber axial stiffness $k_{f}$ and the fiber volume fraction $\mu$ in the EF approach with a constant coefficient $C$, and is independent of the rest of the material parameters such as $z_{f}, \theta_{\max }$ and $\phi$. Eq. 25 indicates that the dimensionless parameters $k_{2}$ and $z_{f}$ take exactly the same value, since $I_{4}=\lambda^{2}$. Eq. 26 implies that the correction $b^{\text {cor }}$ in the AI approach is a function of $b_{0}$ representing the dispersion introduced by fiber angular distribution and $\theta_{\max }$ representing fiber waviness, and is independent of the rest of the material parameters such as $k_{f}$ and $\phi$. When $\theta_{\max }=0^{\circ}$, all fibers are straight and no correction $b^{c o r}$ on the Von Mises parameter is needed.

In order to verify the proposed relationships between the material parameters shown in Eq. 24 to 26, a comprehensive parametric study is conducted. In the parametric study, the ratio of the length and width of the material sample under uniaxial tension is six, and the clamped-clamped boundary condition is used. The simulation results obtained via the EF approach are considered as the reference solution, and the phenomenological parameters used in the AI approach are calibrated to match it. For example, the following optimization process is applied to obtain the relationship between $b^{\text {cor }}$ and $\theta_{\max }$ for a fixed value of $b_{0}$

$$
b^{c o r}=\underset{b^{c o r}}{\arg \min }\left(\left\|\sigma^{c i r c}\left(b^{c o r}\right)-\sigma^{*}\left(\theta_{\max }\right)\right\|_{2}+\left\|\sigma^{\text {axia }}\left(b^{\text {cor }}\right)-\sigma^{*}\left(\theta_{\max }\right)\right\|_{2}\right),
$$

where $\sigma^{\text {circ }}$ and $\sigma^{\text {axia }}$ are obtained from the AI approach, and $\sigma^{*}$ is the corresponding value from the EF approach. 


\section{Results}

The embedded fiber (EF) approach and the angular integration (AI) approach in Sect. 2 follow two different finite element (FE) formulation strategies. The first approach captures the anisotropic behavior of arterial wall at the element level, while the second approach captures the material anisotropy directly at the constitutive level. Correspondingly, a user element subroutine based on Eqs. 6 to 9 and a user material subroutine based on Eqs. 17 to 20 are implemented in an open-source finite element analysis package FEAP (Taylor 2011).

\subsection{Comparison to published experimental data}

In order to demonstrate the accuracy and predictive capability of the proposed $\mathrm{EF}$ and AI approaches, the numerical results obtained via the finite element simulations are compared with the published experimental data of different layers (intima, media and adventitia) of a human abdominal aorta under uniaxial tension (Holzapfel 2006). In the finite element (FE) simulation, all three arterial layers are treated as nearly incompressible material with Poisson's ratio $\nu=0.499$. The 4-node mixed finite element $Q 1 / P 0$ (Taylor 2011) is used throughout the investigation to ensure that the FE formulation is free from locking due to the material nearly incompressibility. The clamped-clamped boundary condition is used to mimic the experimental setup. The experimental data in the original paper were reported in the form of the uniaxial components of the Green-Lagrange strain $\left(E_{11}\right.$ or $\left.E_{22}\right)$ and the 2nd Piola-Kirchhoff stress $\left(S_{11}\right.$ or $\left.S_{22}\right)$. According to the relationship $\lambda=\sqrt{2 E+1}$ and $\sigma=\lambda^{2} S$, the $E-S$ curve is converted into the $\sigma-\lambda$ curve.

Since the fiber volume fraction $\mu$ and the length of individual fiber chain are not reported from the experiment, in the embedded fiber approach, the fiber volume fraction $\mu$ is assumed equal to $1 \%$, and each fiber chain is formed by 10 segments $\left(N_{\text {seg }}=10\right)$ with the segment length $l_{\text {seg }}=50 \mu \mathrm{m}$ that falls in the range $(1 \sim 500$ $\mu \mathrm{m})$ reported in the literature (Annovazzi and Genna 2010). According to Eqs. 22 and 23, the material parameters used in the EF and AI approaches are calibrated and reported in Table 4. Notice that the same group of material parameters are used to fit the experimental data in the circumferential and axial directions.

Figure 7 shows the comparison between the experimental data and the simulation results based on the AI approach. The simulation results match the experimental data well except for the axial sample of the intima layer, in which the two families of fibers are almost aligned with the circumferential direction $\left(\phi= \pm 18.8^{\circ}\right)$. When the axial sample is stretched, initially all fiber chains are under compression (because of the sample transverse contraction) and do not contribute to the material stiffness. At this time the only contribution is from the isotropic matrix component. As the sample stretch increases, fibers are reoriented and transit from the compressive state into the tensile state. Because of the homogenization used in the numerical formulation, this transition happens at the same moment for many fibers, which is manifested by the sudden increase of the slope of the intima $\sigma-\lambda$ curve in Fig. 7b.

In the embedded fiber approach, the random walk algorithm is used to generate the two families of fibers. The relative angle $\theta_{r}$ between neighboring segments 
Table 4 Material parameters used in the FE simulations to fit the experimental data of different layers of the abdominal aorta

\begin{tabular}{c|cccc}
\hline \hline Intima & \multicolumn{4}{l}{} \\
\hline AI & $E=0.30(\mathrm{MPa})$ & $k_{1}=0.1(\mathrm{MPa})$ & $k 2=260$ & $b^{\text {cor }}=-1.62$ \\
\hline EF & $E=0.30(\mathrm{MPa})$ & $k_{f}=4.0 \times 10^{-3}\left(\mathrm{Nmm}^{-1}\right)$ & $z_{f}=260$ & $\theta_{\max }=20^{\circ}$ \\
\hline \hline Media & \multicolumn{3}{c}{} \\
\hline AI & $E=0.05(\mathrm{MPa})$ & $k_{1}=2.0 \times 10^{-2}(\mathrm{MPa})$ & $k 2=5$ & $b^{\text {cor }}=-0.5$ \\
\hline EF & $E=0.05(\mathrm{MPa})$ & $k_{f}=5.5 \times 10^{-4}\left(\mathrm{Nmm}^{-1}\right)$ & $z_{f}=5$ & $\theta_{\max }=20^{\circ}$ \\
\hline \hline Adventitia & & & \\
\hline AI & $E=0.05(\mathrm{MPa})$ & $k_{1}=0.9 \times 10^{-6}(\mathrm{MPa})$ & $k 2=15$ & $b^{\text {cor }}=0.6$ \\
\hline EF & $E=0.05(\mathrm{MPa})$ & $k_{f}=2.5 \times 10^{-8}\left(\mathrm{Nmm}^{-1}\right)$ & $z_{f}=15$ & $\theta_{\max }=10^{\circ}$ \\
\hline \hline
\end{tabular}

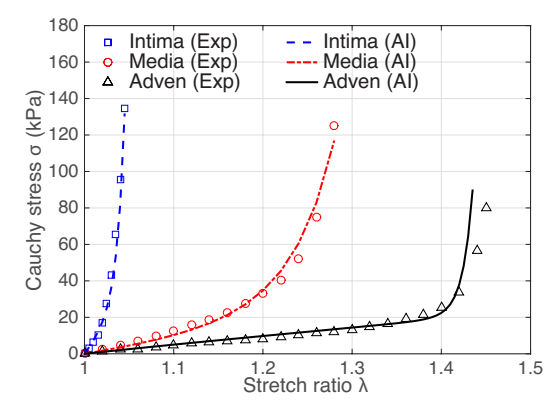

(a) Circumferential direction

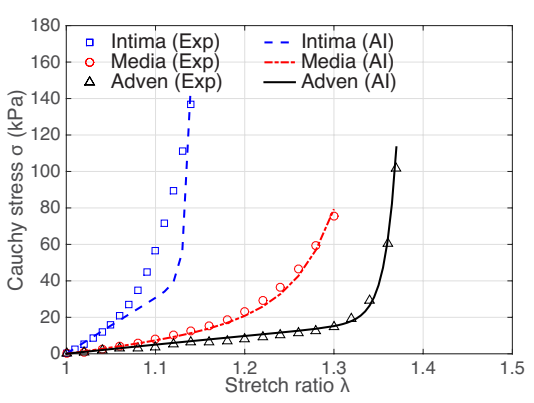

(b) Axial direction

Fig. 7 Comparison of the numerical results obtained via the angular integration (AI) approach with the experimental data (Exp) of different arterial layers

follows the uniform distribution, and the initial angle of each fiber chain $\theta_{0}$ follows the Von Mises distribution with a random starting position. To consider the influence of the stochastic nature of the synthetically generated fiber arrangement on the numerical results, Monte Carlo (MC) simulations are combined with the embedded fiber approach to capture the material behavior in an average sense. Figure 8 shows the distribution of the generated initial angle $\theta_{0}$ of fibers in the intima layer and the resulted geometry of the two families of fibers generated by the random walk algorithm. In the FE simulation, the mesh size $h=l_{\text {seg }}=50 \mu \mathrm{m}$ is used so that the embedded fiber approach provides accurate results. For a detailed discussion about the influence of mesh size on the performance of the embedded fiber approach, the interested reader is referred to Jin and Stanciulescu (2015). From the comparison between the experimental data and the numerical results (mean $\pm \mathrm{SD}$ ) obtained from $500 \mathrm{MC}$ simulations (Fig. 9), it can be seen that the numerical results match the experimental data well in all cases. For the axial sample of the intima layer, since fibers are directly incorporated in the simulation, the transition of fibers from the compressive state into the tensile state happens gradually, thus leading to the smooth increase of the slope of the adventitia $\sigma-\lambda$ curve in Fig. 9b that better captures the behavior observed in experiments. 


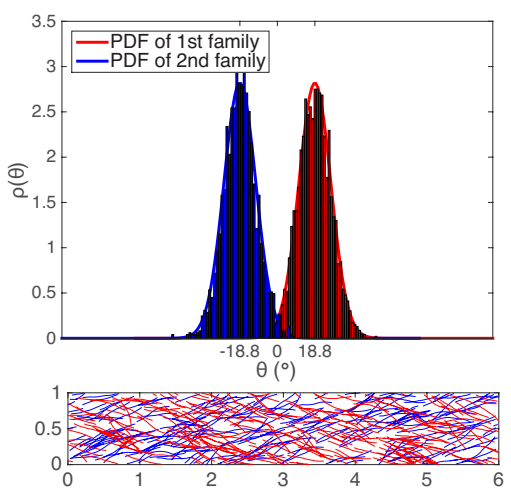

(a) Circumferential sample

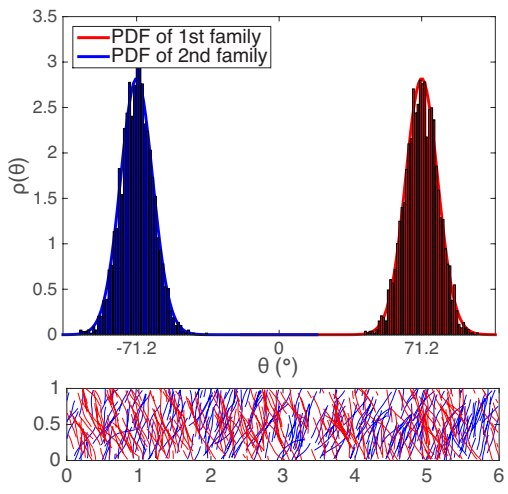

(b) Axial sample

Fig. 8 Distribution of the initial angle $\theta_{0}$ of fibers in the intima layer and the resulted geometry of the two families of fibers generated by the random walk algorithm $\left(\mu=1 \%, \theta_{\max }=20^{\circ}, b=12.732\right)$. For visualization purpose only part of the fibers are shown

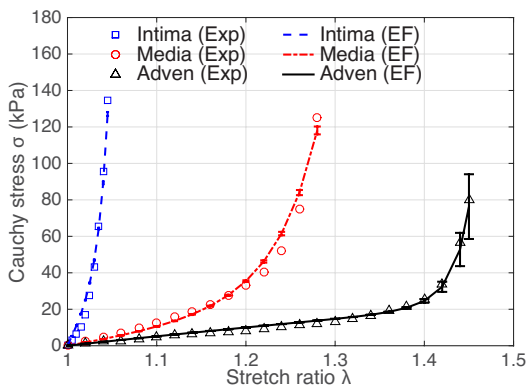

(a) Circumferential direction

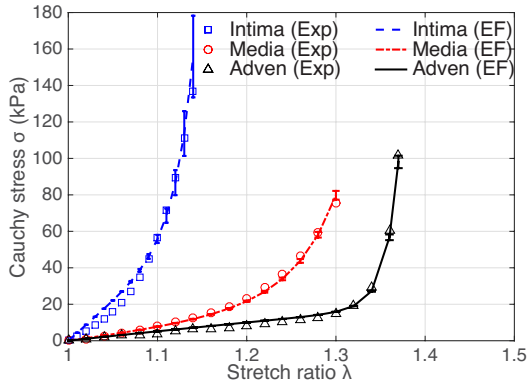

(b) Axial direction

Fig. 9 Comparison of the numerical results obtained via the embedded fiber (EF) approach with the experimental data (Exp) of different arterial layers, $500 \mathrm{MC}$ simulations are carried out to obtain the mean and standard deviation

\subsection{Parametric study}

The relationships between $b^{\text {cor }}$ and $\theta_{\max }$ for different values of $b_{0}$ are obtained according to Eq. 27 and shown in Fig. 10. These relationships are further verified to be independent of the rest of the material parameters including $\mu, k_{f}, z_{f}$ and $\phi$. When $\theta_{\max }=0^{\circ}, b^{\text {cor }}=0$, which is consistent with the fact that when all fibers are straight, no correction on the Von Mises parameter $b$ caused by fiber waviness is needed. Furthermore, it can be seen that as $\theta_{\max }$ increases, the level of material anisotropy decreases because fibers become wavier. Consequently, a larger correction $b^{\text {cor }}$ (absolute value) is needed to properly reflect the influence of fiber waviness on the fiber dispersion (Fig. 10a). When $\theta_{\max }$ becomes large enough $\left(>45^{\circ}\right)$, the material anisotropy is so low that the material behaves almost 
identically in the circumferential and axial directions. Correspondingly, the total value of $b=b_{0}+b^{\text {cor }}$ becomes constant (around 0.5 ) regardless of the value of $b_{0}$ (Fig. 10b), indicating that the material is almost homogeneous.

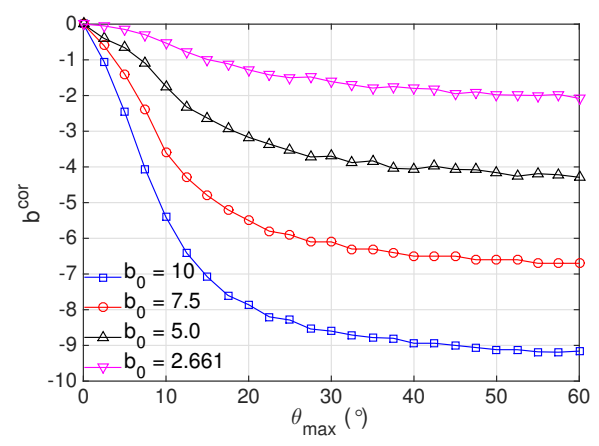

(a) $b^{\text {cor }}-\theta_{\max }$

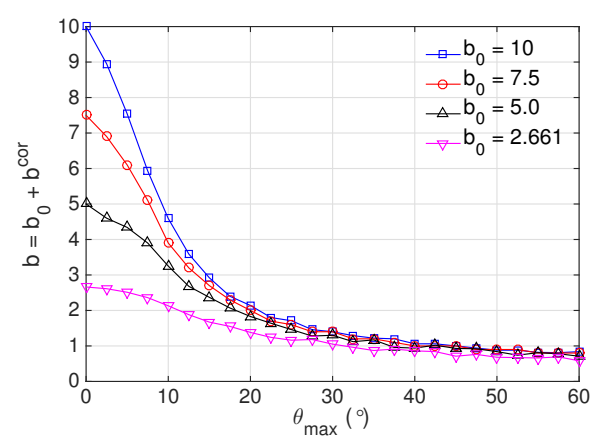

(b) $b=b_{0}+b^{\text {cor }}$

Fig. 10 Relationships between $b^{c o r}$ and $\theta_{\max }$ for different values of $b_{0}$

The relationship between the stress-like parameter $k_{1}$ in the AI approach, and the fiber volume fraction $\mu$ and axial stiffness $k_{f}$ in the EF approach is obtained from a similar optimization process as shown in Eq. 27. From Fig. 11 it can be seen that $k_{1}$ is strictly proportional to $\mu$ and $k_{f}$ with the constant coefficient $C=3.63 \times 10^{3} \mathrm{~mm}^{-1}$ (again, this relationship is verified to be independent of the rest of the material parameters including $\phi, b_{0}, z_{f}$ and $\theta_{\max }$ ). The linear relationship between $k_{1}$ and $\left(k_{f}, \mu\right)$ is expected because of the underlying affine fiber kinematics adopted by the embedded fiber approach, which indicates that the total contribution of fibers is the sum of the contribution from individual fibers.

Based on Eqs 24, 25 and 26, the phenomenological parameters used in the AI approach can be directly obtained from the material parameters used in the EF approach and no optimization is needed. To further verify the accuracy of these relationships, several arbitrary combinations of $\phi, b_{0}, \mu, k_{f}, z_{f}$ and $\theta_{\max }$ that cover large value ranges are tested. In all cases, the numerical results from the two approaches match well in both the circumferential and axial directions under uniaxial tension. Here, only three representative cases are provided for demonstration purposes. The material parameters used are shown in Table 5, and the predicted $\sigma-\lambda$ curves of the AI approach in the circumferential and axial directions are compared with the curves of the EF approach as shown in Fig. 12.

Table 5 Material parameters used in the AI (predicted) and EF approaches based on Eqs 24, 25 and 26

\begin{tabular}{|c|c|c|c|c|c|}
\hline \multicolumn{2}{|c|}{ Case 1} & \multicolumn{2}{|c|}{ Case 2} & \multicolumn{2}{|c|}{ Case 3} \\
\hline \multicolumn{2}{|c|}{$\overline{c \phi}=10^{\circ}, b_{0}=10$} & \multicolumn{2}{|c|}{$\bar{\phi} \phi=37.8^{\circ}, b_{0}=2.661$} & \multicolumn{2}{|c|}{$\overline{\phi \phi}=37.8^{\circ}, b_{0}=2.661$} \\
\hline AI (predicted) & $\mathrm{EF}$ & AI (predicted) & $\mathrm{EF}$ & AI (predicted) & $\mathrm{EF}$ \\
\hline \multirow{2}{*}{$k_{1}=3.63 \times 10^{-4}$} & $k_{f}=1.0 \times 10^{-5}$ & \multirow{2}{*}{$k_{1}=3.99 \times 10^{-2}$} & $k_{f}=5.5 \times 10^{-4}$ & \multirow{2}{*}{$k_{1}=1.45 \times 10^{-1}$} & $k_{f}=1.0 \times 10^{-3}$ \\
\hline & $\mu=1 \%$ & & $\mu=2 \%$ & & $\mu=4 \%$ \\
\hline$k_{2}=4$ & $z_{f}=4$ & $k_{2}=5$ & $z_{f}=5$ & $k_{2}=1.7$ & $z_{f}=1.7$ \\
\hline$b^{\text {cor }}=-8.29$ & $\theta_{\max }=25^{\circ}$ & $b^{\text {cor }}=-1.50$ & $\theta_{\max }=30^{\circ}$ & $b^{\text {cor }}=-0.50$ & $\theta_{\max }=10^{\circ}$ \\
\hline
\end{tabular}

$\dagger k_{1}-\mathrm{MPa} ; k_{f}-\mathrm{Nmm}^{-1}$ 


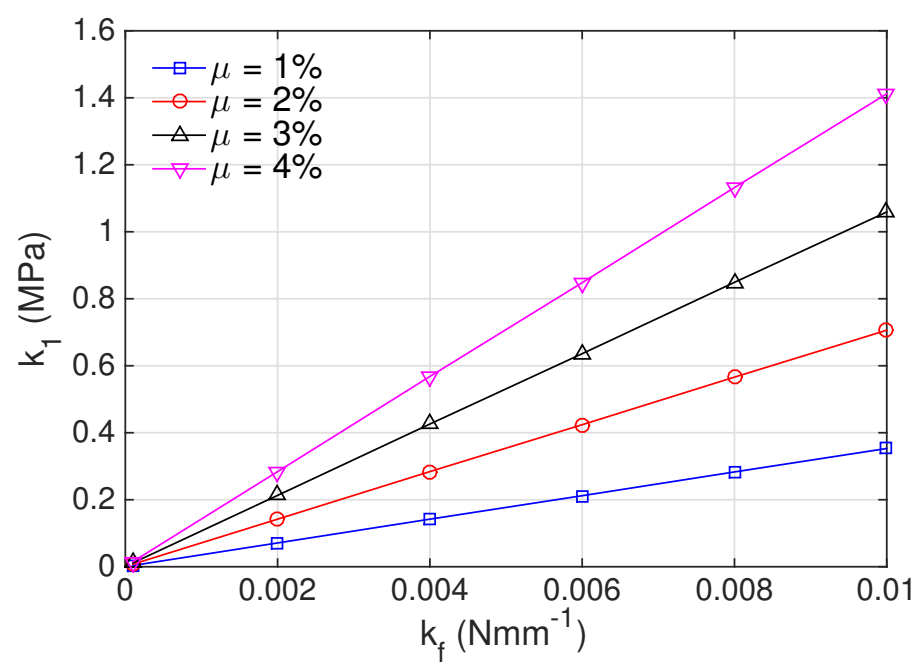

Fig. 11 Linear relationship between $k_{1}$ in the AI approach and $\left(k_{f}, \mu\right)$ in the EF approach

\section{Discussions}

In this paper we present two different modeling approaches, the embedded fiber $(\mathrm{EF})$ approach and the angular integration (AI) approach, to simulate the anisotropic behavior of individual arterial wall layers using layer-specific histological data. Both approaches include the influence of fiber dispersion caused by fiber angular distribution (departure of individual fibers from the mean orientation), and take into consideration the dispersion caused by fiber waviness, which has not been previously considered. By comparing the numerical results with the published experimental data of different layers of a human abdominal aorta, we show that both approaches can successfully capture the material nonlinearity and anisotropy. Furthermore, we establish the relationships between the AI phenomenological parameters and the EF parameters having straightforward geometrical or mechanical interpretations. Through a comprehensive parametric study, we demonstrate the validity and accuracy of these relationships. This study provides valuable insight for the calibration of phenomenological parameters used in the homogenized modeling taking into account the material microscopic arrangement. Moreover, it can facilitate a better understanding of individual arterial wall layers, which will eventually advance the study of the structure-function relationship of the whole arterial wall.

The EF and AI approaches presented in this paper are general in the sense that unlike the generalized structural tensor (GST) method that is only valid for small angular dispersion, they both are valid for any level of fiber angular dispersion. Moreover, both approaches can exclude the contributions from fibers under compression. In the EF approach, this is achieved at each individual fiber via the piecewise fiber strain energy function (Eq. 3). While in the AI approach, the same consideration is adopted by introducing the Heaviside function in the angular integration of the anisotropic strain energy function. Therefore, the two 


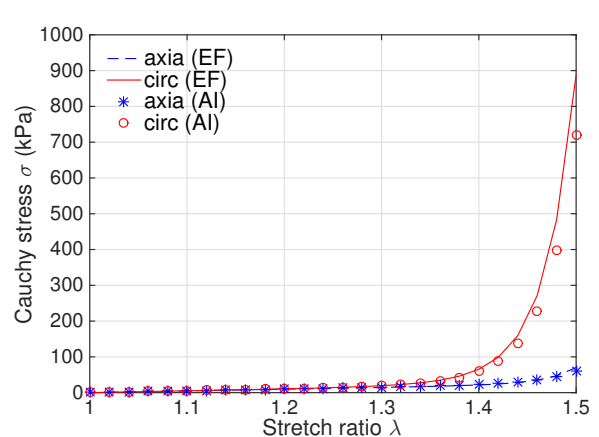

(a) Case 1

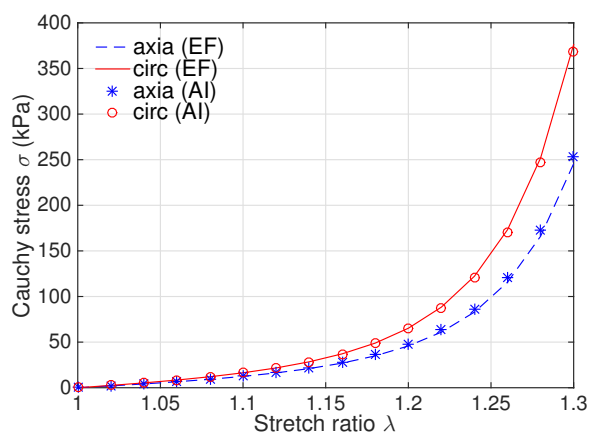

(b) Case 2

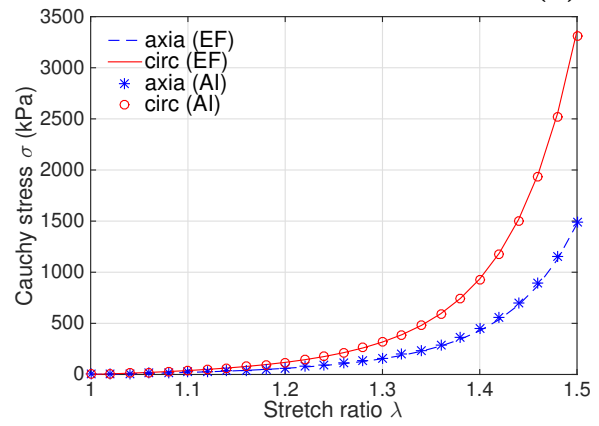

(c) Case 3

Fig. 12 Comparisons of the predicted $\sigma-\lambda$ curves of the AI approach and the curves of the EF approach using the material parameter relationships based on Eqs 24, 25 and 26

approaches can avoid the problem of non-tensile fiber loading present in the GST method.

The finite element (FE) formulations of the EF and AI approaches adopt different ways to capture the material anisotropy. In the EF approach, the anisotropy is captured at the element level by directly incorporating the microscopic arrangement of fibers in the FE mesh. The influence of the material microscopic parameters is implicitly reflected in the element residual and stiffness matrix. Comparing with a standard FE formulation, this approach requires two preprocessing steps, generation of fiber microscopic arrangement via the random walk algorithm and evaluations of element interpolation functions at every fiber segment endpoint. On the other hand, the AI approach directly considers the material anisotropy at the constitutive level by introducing two anisotropic terms in the total strain energy function. Therefore, the FE formulation of this approach is very straightforward. Since the strain energy function is expressed in the integral form, a quadrature rule is needed to calculate the $2 \mathrm{nd} \mathrm{P}-\mathrm{K}$ stress and the material elasticity tensor.

The layer-specific histological data obtained from the experiment include the mean fiber orientations of the two families of fibers $\pm \phi$ and the standard deviations (SD). The EF and AI approaches both use this information, therefore improving the accuracy and predictive capabilities of the numerical modeling. Comparing with existing methods, the two approaches can further take into consideration 
the dispersion caused by fiber waviness, which has not been achieved previously. In the EF approach, fiber waviness is included by introducing the relative angle $\theta_{r}$ between neighbouring segments. While in the AI approach, fiber waviness is considered via the correction term $b^{\text {cor }}$.

Through the comprehensive parametric study, three relationships between the material parameters used in the AI and EF approaches are established:

(1) the relationship between the stress-like parameter $k_{1}$ with the fiber axial stiffness $k_{f}$ and the fiber volume fraction $\mu$;

(2) the relationship between the dimensionless parameters $k_{2}$ and $z_{f}$;

(3) the relationship between the correction term $b^{\text {cor }}$ and the bound of the relative angle $\theta_{\max }$.

Although no rigorous mathematical proof is provided due to the complexity of the problem, these relationships are verified to be true in a large range of random combinations of material parameters. The linear relationship between $k_{1}$ and $\left(k_{f}\right.$, $\mu$ ) is expected, since the EF and AI approaches are both based on the affine fiber kinematics in which the superpostion principle is valid. Due to the same exponential form of the anisotropic strain energy (Eq. 12) in the AI approach and the fiber strain energy (Eq. 3) in the EF approach, the dimensionless $k_{2}$ and $z_{f}$ always take the same value. The relationship between $b^{\text {cor }}$ and $\theta_{\max }$ implies that as $\theta_{\max }$ increases, fibers become wavier and the total level of fiber dispersion increases. Accordingly, the value of $b$ decreases and the material isotropy increases. Especially, when $\theta_{\max }=0$, all fibers are straight and no correction $b^{\text {cor }}$ due to fiber waviness is needed. This is consistent with the original assumption of the structure-based constitutive model (Gasser et al. 2006; Federico and Gasser 2010), in which only the dispersion introduced by departure of fiber from mean orientation (angular distribution) is considered.

In the EF approach, only part of the needed geometric information is provided by the histological images from the experiment, including the mean fiber orientation $\phi$ and standard deviation SD that is equivalent to $b_{0}$. The rest of the material parameters related to the fiber arrangement and mechanical properties are either chosen from the range reported in literature or determined via an optimization process. Recently, many efforts have been devoted to study the mechanical behavior of a single collagen fiber (Annovazzi and Genna 2010; Holzapfel and Ogden 2011, 2013). Also, more detailed geometric information regarding the fiber microscopic arrangement can be acquired via advanced image processing technologies such as confocal reflection imaging (Roeder et al. 2002; Arganda-Carreras et al. 2010) or scanning electron microscopy (SEM) (D'Amore et al. 2010). All these advances make it possible to obtain the material parameters completely from experiments. Based on these material parameters that have straightforward geometrical or physical meaning, phenomenological parameters used in the homogenized modeling can be further decided according to the relationships similar to Eqs 24, 25 and 26 . Therefore, the relatively simple formulation of the homogenized modeling can be maintained. Meanwhile, the difficulties of phenomenological material calibrations typically encountered in the homogenized modeling can be avoided.

In the EF approach, fibers are modeled as truss elements due to two reasons, fiber deformation modes and computational cost. According to Zhang et al. (2013) and D'Amore et al. (2014), fiber stretching is one of the dominant deformation modes and truss elements are very effective in capturing it. Moreover, the primary unknowns of truss elements are the coordinates of the two endpoints that can be 
expressed by the finite element nodal displacements and interpolation functions via the isoparametric relationship (Eq. 2). Thus, the degrees of freedom (DOFs) of the discretized system remain unchanged when fibers are directly included in the FE simulation, and the computational cost is not significantly increased, while the physics is captured well. However, recent studies show that fiber bending may also occur in certain circumstances, for example, see (Holzapfel and Ogden 2011, 2013) for the study of individual biopolymer filament. Fiber bending is not included in the models discussed here and further investigation is needed to accomplish this without significantly increasing the computational cost.

Depending on the different assumptions of the load transfer mechanism inside the material, the affine or nonaffine fiber kinematics can be used. The affine fiber kinematics assumes that the primary load transfer happens between the fiber and the matrix, and the fiber deformation is completely decided by the macroscopic strain field (Lanir 1983; MacKintosh et al. 1995; Agoram and Barocas 2001; Storm et al. 2005). In contrast, the nonaffine fiber kinematics assumes that the primary load transfer happens among fiber chains and each fiber chain deforms independently of the matrix (Chandran and Barocas 2006; Stylianopoulos and Barocas 2007). One of the fundamental assumptions of the EF and AI approaches presented in this paper is the affine fiber kinematics, which is shown to be a valid assumption for pericardial collagenous tissues (Fan and Sacks 2014) and mitral valve anterior leaflet (Lee et al. 2015). On the other hand, there is evidence suggesting that the nonaffine fiber kinematics may be a more realistic assumption for other types of fibrous biomaterial such as bovine annulus fibrous tissue (Head et al. 2003; Chandran and Barocas 2006; Huyghe and Jongeneelen 2012; Lake et al. 2012). How to fully consider the fiber-matrix interaction while adopting the nonaffine fiber kinematics with a reasonable computational cost remains very challenging.

The EF approach uses a random walk algorithm to generate the fiber microscopic arrangement. One of the inputs for this algorithm is that each fiber is formed by a fixed number of segments with the same length. This assumption is a temporary compromise due to lack of reliable information about the fiber length at the microscale. When more accurate fiber topology will become available from experiments via advanced image processing techniques, this assumption can be easily replaced by providing as input the real fiber length.

Lastly, it is worth emphasizing that the relationships between the material parameters used in the EF and AI approaches are only verified for the tensile loading in the circumferential and axial directions. For other loading cases such as shear and bending, whether these relationships are valid still needs further investigation. Also, this paper only focuses on the behavior of individual arterial wall layers. In the next step, we will extend the $2 \mathrm{D}$ formulations presented in this paper into 3D and study the structure-function relationship of arterial walls as a whole.

\section{Acknowledgements}

The authors thank Prof. Jane Grande-Allen for useful discussions and suggestions. This work was partially funded by NSF, grant no 1463432 . 


\section{References}

\section{References}

Agoram B, Barocas VH (2001) Coupled macroscopic and microscopic scale modeling of fibrillar tissues and tissue equivalents. Journal of Biomechanical Engineering 123(4):362-369

Annovazzi L, Genna F (2010) An engineering, multiscale constitutive model for fiber-forming collagen in tension. Journal of Biomedical Materials Research Part A 92(1):254-266

Arganda-Carreras I, Fernández-González R, Muñoz-Barrutia A, Ortiz-DeSolorzano C (2010) 3D reconstruction of histological sections: Application to mammary gland tissue. Microscopy Research and Technique 73(11):1019-1029

Balzani D, Neff P, Schröder J, Holzapfel GA (2006) A polyconvex framework for soft biological tissues. Adjustment to experimental data. International Journal of Solids and Structures 43(20):6052-6070

Barocas VH (2007) Multiscale, Structure-Based Modeling for the Elastic Mechanical Behavior of Arterial Walls. Journal of Biomechanical Engineering 129(4):611

Bonet J, Wood R (2008) Nonlinear Continuum Mechanics for Finite Element Analysis, 2nd edn. Cambridge

Carleton JB, DAmore A, Feaver KR, Rodin GJ, Sacks MS (2015) Geometric characterization and simulation of planar layered elastomeric fibrous biomaterials. Acta Biomaterialia 12:93 101, DOI http://dx.doi.org/10.1016/j.actbio.2014.09.049, URL http://www.sciencedirect.com/science/article/pii/S1742706114004413

Chandran PL, Barocas VH (2006) Affine Versus Non-Affine Fibril Kinematics in Collagen Networks: Theoretical Studies of Network Behavior. Journal of Biomechanical Engineering 128(2):259-270

Chuong CJ, Fung Y (1983) Three-dimensional stress distribution in arteries. Journal of Biomechanical Engineering 105(3):268-274

Cortes DH, Lake SP, Kadlowec JA, Soslowsky LJ, Elliott DM (2010) Characterizing the mechanical contribution of fiber angular distribution in connective tissue: Comparison of two modeling approaches. Biomechanics and Modeling in Mechanobiology 9(5):651-658

D'Amore A, Stella JA, Wagner WR, Sacks M (2010) Characterization of the complete fiber network topology of planar fibrous tissues and scaffolds. Biomaterials 31(20):5345-5354

D'Amore A, Amoroso N, Gottardi R, Hobson C, Carruthers C, Watkins S, Wagner WR, Sacks M (2014) From single fiber to macro-level mechanics: A structural finite-element model for elastomeric fibrous biomaterials. Journal of the mechanical behavior of biomedical materials 39:146-161

Durst CA, Cuchiara MP, Mansfield EG, West JL, Grande-Allen KJ (2011) Flexural characterization of cell encapsulated PEGDA hydrogels with applications for tissue engineered heart valves. Acta biomaterialia 7(6):2467-2476

Fan R, Sacks M (2014) Simulation of planar soft tissues using a structural constitutive model: Finite element implementation and validation. Journal of biomechanics 47(9):2043-2054

Federico S, Gasser TC (2010) Nonlinear elasticity of biological tissues with statistical fibre orientation. Journal of the Royal Society Interface 7(47):955-966 
Federico S, Herzog W (2008) Towards an analytical model of soft biological tissues. Journal of biomechanics 41(16):3309-3313

Fung Y (1967) Elasticity of soft tissues in simple elongation. The American journal of physiology 213(6):1532-1544

Fung Y (1983) On the Foundations of Biomechanics. Journal of Applied Mechanics, Transactions ASME 50(4 b):1003-1009

Fung Y, Fronek K, Patitucci P (1979) Pseudoelasticity of arteries and the choice of its mathematical expression. The American journal of physiology 237(5):H620631

Gasser TC, Ogden RW, Holzapfel GA (2006) Hyperelastic modelling of arterial layers with distributed collagen fibre orientations. Journal of the Royal Society Interface 3(6): 15-35

Hasan A, Ragaert K, Swieszkowski W, Selimović T, Paul A, Camci-Unal G, Mofrad MRK, Khademhosseini A (2014) Biomechanical properties of native and tissue engineered heart valve constructs. Journal of biomechanics 47(9):1949-1963

Head DA, Levine AJ, MacKintosh FC (2003) Deformation of Cross-Linked Semiflexible Polymer Networks. Physical Review Letters 91(10):1081,0211081,024

Heidemann KM, Sharma A, Rehfeldt F, Schmidt CF, Wardetzky M (2015) Elasticity of $3 \mathrm{D}$ networks with rigid filaments and compliant crosslinks. Soft Matter 11(2):343-354

Holzapfel G, Stadler M, Schulze-Bauer C (2002) A layer-specific three-dimensional model for the simulation of balloon angioplasty using magnetic resonance imaging and mechanical testing. Annals of Biomedical Engineering 30(6):753-767, DOI 10.1114/1.1492812, URL http://dx.doi.org/10.1114/1.1492812

Holzapfel GA (2000) Nonlinear Solid Mechanics, a Continuum Approach for Engineering. Wiley

Holzapfel GA (2006) Determination of material models for arterial walls from uniaxial extension tests and histological structure. Journal of Theoretical Biology 238(2):290-302

Holzapfel GA, Ogden RW (2011) On the bending and stretching elasticity of biopolymer filaments. Journal of Elasticity 104(1-2):319-342

Holzapfel GA, Ogden RW (2013) Elasticity of biopolymer filaments. Acta biomaterialia 9(7):7320-7325

Holzapfel GA, Gasser TC, Ogden RW (2000) A new constitutive framework for arterial wall mechanics and a comparative study of material models. Journal of Elasticity 61(1-3):1-48

Holzapfel GA, Sommer G, Regitnig P (2004) Anisotropic mechanical properties of tissue components in human atherosclerotic plaques. Journal of Biomechanical Engineering 126(5):657-665

Holzapfel GA, Sommer G, Gasser CT, Regitnig P (2005) Determination of layerspecific mechanical properties of human coronary arteries with nonatherosclerotic intimal thickening and related constitutive modeling. American Journal of Physiology - Heart and Circulatory Physiology 289(5 58-5):H2048-H2058

Huisman EM, van Dillen T, Onck PR, Van der Giessen E (2007) Three-dimensional cross-linked F-actin networks: Relation between network architecture and mechanical behavior. Physical Review Letters 99(20)

Huyghe JM, Jongeneelen CJM (2012) 3D non-affine finite strains measured in isolated bovine annulus fibrosus tissue samples. Biomechanics and Modeling in 
Mechanobiology 11(1-2):161-170

Jin T, Stanciulescu I (2015) Numerical simulation of fibrous biomaterials with randomly distributed fiber network structure. Biomechanics and Modeling in Mechanobiology pp 1-14, DOI 10.1007/s10237-015-0725-6, URL http://dx.doi.org/10.1007/s10237-015-0725-6

Lake SP, Hadi MF, Lai VK, Barocas VH (2012) Mechanics of a fiber network within a non-fibrillar matrix: Model and comparison with collagen-agarose cogels. Annals of Biomedical Engineering 40(10):2111-2121

Lanir Y (1983) Constitutive equations for fibrous connective tissues. Journal of biomechanics 16(1):1-12

Lee CH, Zhang W, Liao J, Carruthers CA, Sacks JI, Sacks MS (2015) On the presence of affine fibril and fiber kinematics in the mitral valve anterior leaflet. Biophysical Journal 108(8):2074-2087

Liu Q, Lu Z, Hu Z, Li J (2013) Finite element analysis on tensile behaviour of 3D random fibrous materials: Model description and meso-level approach. Materials Science and Engineering A 587:36-45

MacKintosh FC, Käs J, Janmey PA (1995) Elasticity of semiflexible biopolymer networks. Physical Review Letters 75(24):4425-4428

Martins PALS, Jorge RMN, Ferreira AJM (2006) A comparative study of several material models for prediction of hyperelastic properties: Application to siliconerubber and soft tissues. Strain 42(3):135-147

Pandolfi A, Vasta M (2012) Fiber distributed hyperelastic modeling of biological tissues. Mechanics of Materials 44:151-162

Roeder BA, Kokini K, Sturgis JE, Robinson JP, Voytik-Harbin SL (2002) Tensile mechanical properties of three-dimensional type I collagen extracellular matrices with varied microstructure. Journal of Biomechanical Engineering 124(2):214222

Sander EA, Stylianopoulos T, Tranquillo RT, Barocas VH (2009) Image-based multiscale modeling predicts tissue-level and network-level fiber reorganization in stretched cell-compacted collagen gels. Proceedings of the National Academy of Sciences 106(42):17,675-17,680, DOI 10.1073/pnas.0903716106, URL http://www.pnas.org/content/106/42/17675.abstract, http://www.pnas.org/content/106/42/17675.full.pdf

Sarma PA, Pidaparti RM, Moulik PN, Meiss RA (2003) Non-linear material models for tracheal smooth muscle tissue. Bio-Medical Materials and Engineering 13(3):235-245

Shahsavari AS, Picu RC (2013) Size effect on mechanical behavior of random fiber networks. International Journal of Solids and Structures 50(20-21):3332-3338

Spanos P, Esteva M (2009) Effect of stochastic nanotube waviness on the elastic and thermal properties of nanocomposites by fiber embedment in finite elements. Journal of Computational and Theoretical Nanoscience 6(10):2317-2333

Spencer A (ed) (1984) Continuum Theory of the Mechanics of Fiber-reinforced Composites. Springer

Storm C, Pastore JJ, MacKintosh FC, Lubensky TC, Janmey PA (2005) Nonlinear elasticity in biological gels. Nature 435(7039):191-194

Stylianopoulos T, Barocas VH (2007) Volume-averaging theory for the study of the mechanics of collagen networks. Computer Methods in Applied Mechanics and Engineering 196(31-32):2981-2990 
Taylor R (2011) FEAP, a Finite Element Analysis Program: Version 8.3 User Manual. University of California, Berkeley. Dept. of Civil and Environmental Engineering

Vawter D, Fung Y, West J (1979) Constitutive Equation of Lung Tissue Elasticity. American Society of Mechanical Engineers, Applied Mechanics Division, AMD 32:135-138

Wang Y, Son S, Swartz SM, Goulbourne NC (2012) A mixed von Mises distribution for modeling soft biological tissues with two distributed fiber properties. International Journal of Solids and Structures 49(21):2914-2923

Wilber JP, Walton JR (2002) The Convexity Properties of a Class of Constitutive Models for Biological Soft Issues. Mathematics and Mechanics of Solids $7(3): 217-235$

Zhang L, Lake SP, Lai VK, Picu CR, Barocas VH, Shephard MS (2013) A coupled fiber-matrix model demonstrates highly inhomogeneous microstructural interactions in soft tissues under tensile load. Journal of Biomechanical Engineering 135(1):011,008-011,008 